\title{
Bioinformatic analysis of glycoside hydrolases in the proteomes of mesophilic and thermophilic Actinobacteria
}

\begin{abstract}
Petroleum reserves are rapidly depleting and alternative renewable sources of energy need to be developed to meet the energy demands of the planet. Lignocellulose has been recognized as a highly promising and renewable resource for the development of clean energy. Thermophilic microbes and thermostable enzymes are being sought for biological conversion of lignocellulose into biofuels. The phylum Actinobacteria includes several efficient cellulose-degrading microorganisms. Genomes of several Actinobacteria have been completely sequenced and deposited in public databases, which are a great resource for uncovering new enzymes and targets for biotechnology. We searched the predicted proteomes of 69 Actinobacteria for the homologs of 20 glycoside hydrolase families relevant to lignocellulose degradation and identified 589 glycoside hydrolase homologs. We analyzed (1) the distribution of the glycoside hydrolase homologs across mesophilic and thermophilic Actinobacteria (2), the domain architecture of cellulases (from GH5 and GH6 families) and xylanases (from GH10 and GH11 families) from mesophilic and thermophilic Actinobacteria, and (3) asymmetric amino acid substitutions between mesophilic and thermophilic glycoside hydrolases. Overall, our data provide new insights into the distribution of different glycoside hydrolases in Actinobacteria as well as into the thermostability features of cellulases and xylanases from Actinobacteria. Our findings provide a basis for genetic engineering of glycoside hydrolases as well as new targets for biotechnology.
\end{abstract}

Keywords: thermophiles, enzymes, cellulases, xylanases, biofuel, lignocellulose, genome, proteome
Volume 5 Issue 3 - 2017

\section{Kip A Teegardin, Steven James, Ravi D Barabote}

Department of Biological Sciences, University of Arkansas, USA

Correspondence: Ravi D. Barabote, Department of Biological Sciences, University of Arkansas, Fayetteville, AR 7270I, USA,. Tel (479) 5752475, Fax (479) 57540 I0, Email barabote@uark.edu

Received: January 30, 2017 | Published: March 16, 2017
Abbreviations: GH, glycoside hydrolases; CBM, carbohydrate binding module; OGT, optimal growth temperature

\section{Introduction}

Petroleum fuels are finite and non-renewable and they pose a significant concern for global climate, sustainability, and international security. ${ }^{1}$ Alternative renewable sources of energy are urgently needed to meet the current global challenges. Plants are the most abundant source of renewable carbon on Earth. Plant cell wall (lignocellulose) can be used for the production of renewable, sustainable, and environmentally -clean biofuels. ${ }^{2}$ Lignocellulose is mainly composed of polymers of sugars (cellulose and hemicellulose) and phenolic units (lignin). While complex lignocellulose can be converted into liquid fuels thermo-chemically, biological transformation of lignocellulosic polysaccharides using microorganisms and microbial enzymes is an economical and environmentally benign process for sustainable production of biofuels. ${ }^{3,4}$ Several microorganisms produce glycoside hydrolase enzymes such as cellulases and xylanases that break down cellulose and xylan (hemicellulose), respectively. ${ }^{5}$ Efficient lignocellulose-degrading microorganisms and catalytically- superior cellulases and xylanases are of very high value in the bioconversion of lignocellulose into biofuels. ${ }^{6,7}$

Actinobacteria are a phylum of Gram-positive bacteria that are found abundantly in soil. ${ }^{8}$ They include some of the most prolific lignocellulose-degrading bacteria. ${ }^{9}$ Actinobacteria include both mesophilic and thermophilic members. Many new Actinobacteria continue to be isolated and sequenced in bioprospecting studies aimed at identifying new biotechnological targets. ${ }^{10}$ Growing number of completely sequenced genomes are being steadily deposited in public databases, which provide an expanding resource for discovering novel targets for biotechnology. Systematic bioinformatic mining of the genomes and predicted proteomes of sequenced Actinobacteria has the potential to reveal novel insights into lignocellulose-degrading enzymes for bioenergy applications. ${ }^{11}$

Thermophilic microbes and thermostable enzymes are most useful for the development of cost- effective, industrial scale technologies. ${ }^{12}$ Thermostability of enzymes increases their shelf life, reduces reaction times, improves industrial productivity, and lowers manufacturing costs. ${ }^{12}$ Thus, enzyme thermostability is a highly desirable property for industrial enzymatic deconstruction of lignocellulose. Valuable insights can be gleaned about factors that contribute to thermostability by performing comparative analysis of amino acid sequences of proteins from mesophilic and thermophilic organisms. ${ }^{13}$ Such insights can be exploited for designing and genetically engineering enhanced enzymes for industrial applications.

In this study, we systematically analyzed the predicted proteomes of 69 Actinobacteria for homologs of glycoside hydrolase enzymes that are relevant to lignocellulose degradation. We analyzed the distribution of the homologs across the phylum. We identified homologs from mesophilic and thermophilic Actinobacteria and analyzed their domain architecture to decipher thermophilic patterns. Finally, we analyzed the amino acid sequences of cellulases and xylanases from mesophilic and thermophilic Actinobacteria and identified asymmetric amino acid substitution patterns in the thermophilic enzymes. 


\section{Methodology}

Predicted proteomes of known lignocellulose-degrading Actinobacteria were obtained from NCBI (ftp://ftp.ncbi.nlm. nih.gov/). Optimal growth temperature (OGT) information was obtained through literature. Organisms were classified as mesophilic $\left(\mathrm{OGT}<40^{\circ} \mathrm{C}\right)$ or thermophilic $\left(\mathrm{OGT}>40^{\circ} \mathrm{C}\right)$. Glycoside hydrolase families that contain lignocellulose degradation enzymes were identified from the CAZy database. ${ }^{14}$ Representative Actinobacterial sequences from the CAZy families were used to identify homologs in the proteomes of the Actinobacteria using BLAST. ${ }^{15}$ Domains in the glycoside hydrolase proteins were identified using the NCBI's CDD- search tool. ${ }^{16}$ Amino acid substitutions between homologs of mesophilic and thermophilic Actinobacteria were identified using multiple alignments as described previously. ${ }^{17}$ Briefly, for each GH family, orthologs from mesophilic and thermophilic Actinobacteria were aligned using CLUSTAL. ${ }^{18,19}$ Each substitution was counted only once per position in the alignment. For each amino acid substitution pair (e.g., AMBT and ATBM where A and B represent amino acids and the subscripts $\mathrm{M}$ and $\mathrm{T}$ represent mesophilic and thermophilic organisms, respectively), the total number of substitutions over the entire alignment was summed and the percentage of each substitution within the pair was calculated. Statistical significance ( $p$-value) of asymmetric amino acid substitutions between the two groups of organisms was calculated using a binomial function. The asymmetry (i.e., bias) in AMBT and ATBM substitutions was considered significant if their p-value was below the threshold.

\section{Results and discussion}

\section{Distribution of glycoside hydrolases in Actinobacteria}

We identified a total of 1133 Actinobacteria in the NCBI database. Of these, genomes of only $236(21 \%)$ Actinobacteria have been completed sequenced. Within the 236 sequenced Actinobacteria, we identified $69(29 \%)$ organisms that have been described in literature to have cellulolytic activity. We analyzed the predicted proteomes of the 69 Actinobacteria for the presence of glycoside hydrolases relevant to lignocellulose degradation. In addition, we collected information on their optimal growth temperature for each organism from literature. Using the CAZy database, we identified 20 glycoside hydrolase families that contain enzymes known to hydrolyze various plant cell wall polysaccharides. ${ }^{14}$ A total of 589 glycoside hydrolase homologs were identified in the proteomes of the 69 Actinobacteria (Table 1). Of the 69 Actinobacteria,". 61 organisms are mesophilic and only 8 are thermophilic. This highlights the need to sequence more thermophilic Actinobacteria.

Table I Summary of the analysis of Actinobacteria used in this study

\begin{tabular}{|c|c|c|c|}
\hline Organism name & OGT $\left({ }^{\circ} \mathrm{C}\right)$ & \# of GH families & Total GH homologs \\
\hline Acidothermus cellulolyticus I IB ATCC 43068 & 55 & 7 & 12 \\
\hline Actinosynnema mirum DSM 43827 & 28 & 9 & 30 \\
\hline Amycolatopsis mediterranei $\$ 699$ & 26 & 13 & 37 \\
\hline Amycolatopsis mediterranei U32 & 26 & 12 & 33 \\
\hline Bifidobacterium adolescentis 15703 & 50 & 3 & 4 \\
\hline Bifidobacterium animalis ADO I I & 37 & 1 & 1 \\
\hline Bifidobacterium animalis ATCC 25527 & 37 & I & 1 \\
\hline Bifidobacterium animalis B420 & 37 & I & 1 \\
\hline Bifidobacterium animalis $B b / 2$ & 37 & 2 & 2 \\
\hline Bifidobacterium animalis Bi-04 & 37 & 1 & 1 \\
\hline Bifidobacterium animalis Bi-07 & 37 & 1 & 2 \\
\hline Bifidobacterium animalis $\mathrm{BLCl}$ & 37 & I & 1 \\
\hline Bifidobacterium animalis CNCM I-2494 & 37 & 2 & 3 \\
\hline Bifidobacterium animalis DSM 10140 & 37 & I & 1 \\
\hline Bifidobacterium animalis V9 & 37 & 1 & 1 \\
\hline Bifidobacterium bifidum PRL20IO & 37 & 1 & 2 \\
\hline Bifidobacterium bifidum SI 7 & 37 & 1 & 2 \\
\hline Bifidobacterium breve ACS-07I-V-Sch8b & 37 & 2 & 2 \\
\hline Bifidobacterium breve UCC2003 & 37 & 0 & 0 \\
\hline Bifidobacterium dentium BdI & 29 & 2 & 7 \\
\hline Bifidobacterium Longum $157 \mathrm{~F}$ & 34 & 2 & 6 \\
\hline Bifidobacterium Longum BBMN68 & 34 & 1 & 3 \\
\hline Bifidobacterium Longum DJOIOA & 34 & 1 & 2 \\
\hline Bifidobacterium Longum F8 & 34 & 1 & 1 \\
\hline Bifidobacterium Longum JCM I2I7 & 34 & 2 & 5 \\
\hline Bifidobacterium Longum JCM I 222 ,ATCC 15697 & 34 & 2 & 3 \\
\hline
\end{tabular}


Table Continued....

\begin{tabular}{|c|c|c|c|}
\hline Organism name & OGT $\left({ }^{\circ} \mathrm{C}\right)$ & \# of GH families & Total GH homologs \\
\hline Bifidobacterium Longum JDM30I & 34 & 2 & 5 \\
\hline Bifidobacterium Longum KACC 91563 & 34 & 2 & 4 \\
\hline Bifidobacterium Longum NCC2705 & 34 & 2 & 5 \\
\hline Cellulomonas fimi ATCC 484 & 40 & 11 & 34 \\
\hline Cellulomonas flavigena DSM 20109 & 30 & 9 & 37 \\
\hline Cellvibrio gilvus ATCC $13 / 27$ & 25 & 9 & 20 \\
\hline Clavibacter michiganensis NCPPB 382 & 37 & 3 & 7 \\
\hline Clavibacter michiganensis sepedonicus & 37 & 1 & 2 \\
\hline Jonesia denitrificans DSM 20603 & 37 & 9 & 13 \\
\hline Micrococcus luteus & 37 & 0 & 0 \\
\hline Micromonospora aurantiaca ATCC 27029 & 27 & 10 & 22 \\
\hline Modestobacter marinus $\mathrm{BC} 50 \mathrm{I}$ & 28 & 0 & 0 \\
\hline Mycobacterium abscessus & 30 & 2 & 2 \\
\hline Mycobacterium avium 104 & 37 & 3 & 3 \\
\hline Mycobacterium avium $\mathrm{K}-\mathrm{IO}$ & 37 & 3 & 3 \\
\hline Mycobacterium bovis AF2 I 22/97 & 35 & 4 & 5 \\
\hline Mycobacterium bovis BCG str. Mexico & 35 & 3 & 3 \\
\hline Mycobacterium bovis Pasteur II 73P2 & 35 & 3 & 3 \\
\hline Mycobacterium bovis Tokyo I 72 & 35 & 3 & 3 \\
\hline Mycobacterium gilvum PYR-GCK & 30 & 3 & 3 \\
\hline Mycobacterium marinum & 37 & 2 & 2 \\
\hline Mycobacterium smegmatis MC2 I55 & 30 & 3 & 4 \\
\hline Rhodococcus erythropolis PR4 PR4 (= NBRC 100887) & 25 & 2 & 2 \\
\hline Rhodococcus opacus B4 & 27 & 2 & 2 \\
\hline Saccharomonospora glauca & 45 & 0 & 0 \\
\hline Saccharomonospora viridis DSM 43017 & 55 & 2 & 2 \\
\hline Streptomyces avermitilis MA-4680 & 32 & 9 & 19 \\
\hline Streptomyces bingchenggensis BCW-I & 28 & 12 & 47 \\
\hline Streptomyces cattleya DSM 46488,8057 & 34 & 4 & 12 \\
\hline Streptomyces clavuligerus & 28 & 0 & 0 \\
\hline Streptomyces coelicolor A3(2) & 28 & 11 & 20 \\
\hline Streptomyces flavogriseus ATCC 33331 & 28 & 11 & 19 \\
\hline Streptomyces hygroscopicus jinggangensis 5008 & 35 & 9 & 13 \\
\hline Streptomyces pristinaespiralis & 28 & 0 & 0 \\
\hline Streptomyces scabiei 87.22 & 27 & 10 & 27 \\
\hline Streptomyces sirex $A A 3$ & 28 & 8 & 9 \\
\hline Streptomyces sviceus & 28 & 1 & 1 \\
\hline Streptomyces violaceusniger Tu 4 II3 & 28 & 10 & 22 \\
\hline Streptosporangium roseum DSM 43021 & 28 & 9 & 14 \\
\hline Thermobifida fusca YX & 55 & 8 & 12 \\
\hline Thermobispora bispora 43833 & 55 & 8 & 10 \\
\hline Thermomonospora curvata 43 I 83 & 55 & 2 & 3 \\
\hline Xylanimonas cellulosilytica DSM 15894 & 30 & 7 & 12 \\
\hline
\end{tabular}

OGT $\left({ }^{\circ} \mathrm{C}\right.$ ), optimal growth temperature (degrees Celsius); $\mathrm{GH}$, glycoside hydrolase 
We analyzed the relationship between optimal growth temperature and glycoside hydrolases encoded in the proteomes of the Actinobacteria (Figure 1). In general, there was very poor correlation $(\mathrm{R} 2<0.1)$ between optimal growth temperature and glycoside hydrolase content of the proteomes. However, this may be partly due to the overrepresentation of mesophilic Actinobacteria in the dataset. The 61 mesophilic Actinobacteria encoded between 0 and 13 glycoside hydrolase families with an average of $4.0 \pm 3.9$, while they encoded between 0 and 47 homologs of glycoside hydrolases with an average of $8.4 \pm 10.9$. The 8 thermophilic Actinobacteria encoded between 0 and 11 glycoside hydrolase families with an average of $5.1 \pm 3.9$, while they encoded between 0 and 34 homologs of glycoside hydrolases with an average of $9.6 \pm 10.9$. There were no statistically significant differences in the distribution of glycoside hydrolases between mesophilic and thermophilic Actinobacteria. However, substantially greater numbers of thermophilic Actinobacteria need to be sequenced before deciphering any underlying biases between the two groups of Actinobacteria.

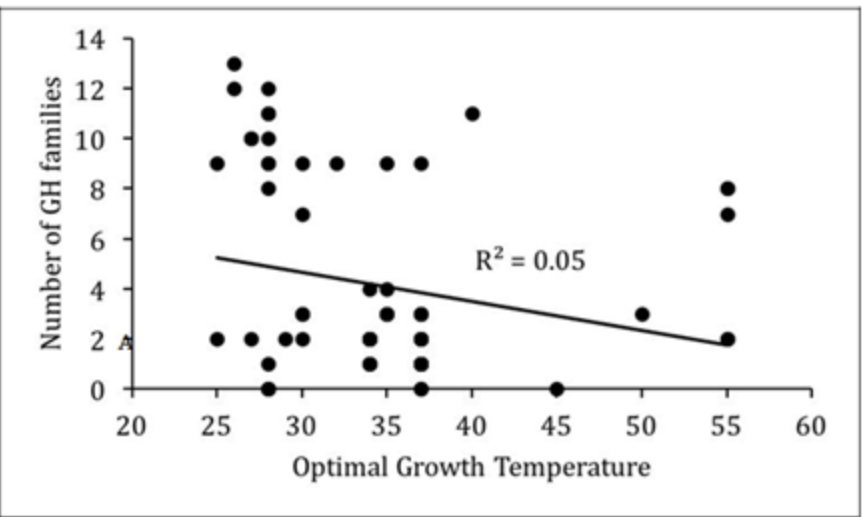

A

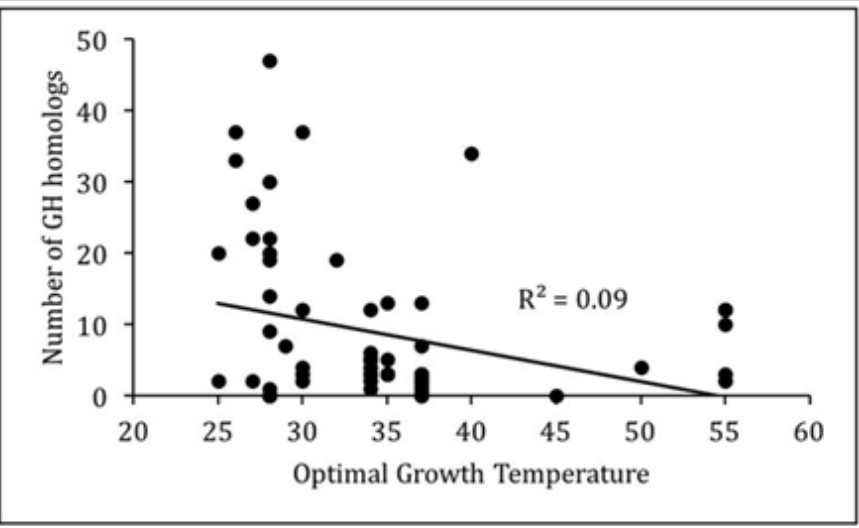

Figure I Relationship between optimal growth temperature and glycoside hydrolases in Actinobacteria.

(A) Scatter plot of number of glycoside hydrolases $(\mathrm{GH})$ families versus optimal growth temperature.

(B) Scatter plot of number GH homologs versus optimal growth temperature. Best-fit line with R-squared value is shown.

We analyzed relative abundances of the 20 glycoside hydrolase families across Actinobacteria (Figure 2). The data show that GH5 was the most highly represented family in the Actinobacteria.
It was the only family that was found in majority (70\%) of the organisms analyzed. The GH6 and GH43 families were the next most represented families and were found in $48 \%$ of the Actinobacteria. The GH5 family is known to contain cellulose- and hemicellulosedegrading enzymes, while the GH6 family contains cellulases and the GH43 family contains hemicellulases. ${ }^{14}$ The GH45, GH51, and

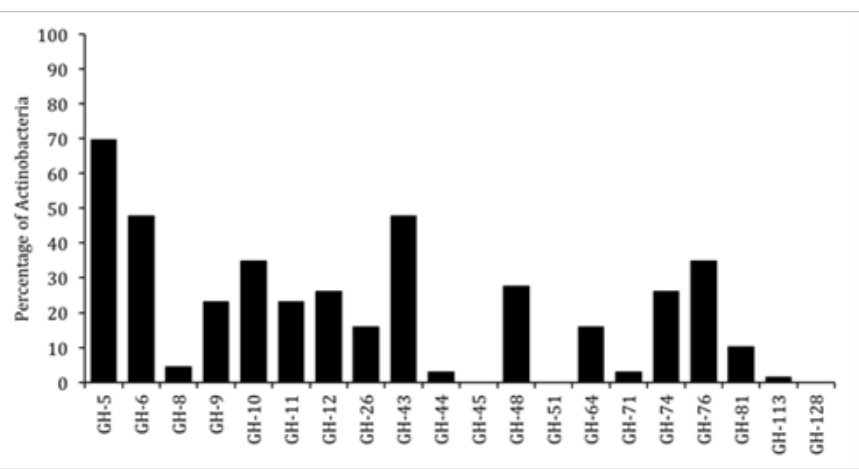

GH128 families were not represented in any of the Actinobacteria in our dataset. Other GH families showed intermediate representation.

Figure 2 Relative abundance of glycoside hydrolase (GH) families in Actinobacteria. Percentage of Actinobacteria containing homologs of the different $\mathrm{GH}$ families are plotted.

\section{Domain architecture of glycoside hydrolases in Actinobacteria}

To minimize over-representation of mesophilic Actinobacteria in the dataset, we selected one representative species per genus and also retained saprophytic free-living bacteria while removing animal and human pathogens. This yielded a more balanced set of Actinobacteria (6 thermophiles and 8 mesophiles). We focused our analysis on four GH families - cellulases from GH5 and GH6 families and xylanases from GH10 and GH11 families. There were 113 glycoside hydrolases from the four families across the 14 Actinobacteria (Table 2). There were 77 homologs in the 8 mesophilic bacteria, and 36 homologs in the 6 thermophilic bacteria. Six organisms contained representatives from all four families, while five organisms contained representatives from only three families and two organisms contained homologs from just one family. We analyzed the domain architecture of the 113 glycoside hydrolases using the NCBI's CDD-search tool. ${ }^{16}$ At least five different types of carbohydrate binding modules (CBMs - CBM2, CBM-3, CBM-X2, CBM-9, and CBM-4-9) were found fused to the catalytic domains of glycoside hydrolases (Table 3). Further analysis revealed a bias in the presence and location of certain CBMs. For example, CBM-2 was found fused on the $\mathrm{C}$-terminal side of the catalytic domain in all four glycoside hydrolase families, while it was found on the $\mathrm{N}$-terminal side of the catalytic domain in GH5 and GH6 cellulases. CMB -3 was only found in homologs from thermophilic Actinobacteria, and it always occurred C-terminal to the catalytic hydrolase domain. CBM- 9 and CMB-4-9 were found attached to only GH10 xylanases. CBM-9 occurred C- terminal to the catalytic domain, while CBM-4-9 was found on the N-terminal side of the hydrolase domain. CBM-X2 was found only in GH5 hydrolases from mesophilic Actinobacteria and was found C-terminal to the hydrolase domain. These data suggest that there are positional constraints for CBM domains in glycoside hydrolases. Certain domains may be required for the functioning and stability of the enzymes, while others may be specific to the substrates hydrolyzed by the associated catalytic domains. 
Table 2 Distribution of glycoside hydrolases in mesophilic and thermophilic Actinobacteria

\begin{tabular}{llllll}
\hline Organism name & OGT & GH5 & GH6 & GHIO & GHII \\
\hline (A) Mesophilic Actinobacteria & 28 & 5 & 3 & 4 & I \\
Actinosynnema mirum DSM 43827 & 26 & 4 & 2 & 7 & I \\
Amycolatopsis mediterranei S699 & 25 & 2 & 4 & 6 & 0 \\
Cellvibrio gilvus ATCC I3 I 27 & 37 & 0 & 2 & 4 & I \\
Jonesia denitrificans DSM 20603 & 27 & 4 & 2 & 4 & I \\
Micromonospora aurantiaca ATCC 27029 & 28 & I & 3 & 2 & 2 \\
Streptomyces coelicolor A3(2) & 28 & I & 3 & I & 0 \\
Streptosporangium roseum DSM 4302I & 30 & 0 & 2 & 4 & I \\
Xylanimonas cellulosilytica DSM I5894 & & & & & \\
(B) Thermophilic Actinobacteria & 55 & 2 & 2 & 2 & 0 \\
Acidothermus cellulolyticus IIB & 40 & 0 & 6 & 8 & I \\
Cellulomonas fimi ATCC 484 & 55 & 0 & 0 & I & 0 \\
Saccharomonospora viridis DSM 430 I7 & 55 & 2 & 2 & 2 & I \\
Thermobifida fusca YX & 55 & I & 2 & 2 & I \\
Thermobispora bispora DSM 43833 & 55 & 0 & I & 0 & 0 \\
Thermomonospora curvata DSM 43 I83 & & & & \\
\hline
\end{tabular}

Table 3 Domain architecture of glycoside hydrolases in Actinobacteria

\section{C-Terminal domain}

\begin{tabular}{|c|c|c|c|c|c|c|c|c|c|c|c|}
\hline (A) Mesophiles & & GH5 & GH6 & GHIO & GHII & CBM_2 & CBM_3 & CBM_X2 & CBM_9 & CBM_4_9 & No CBM \\
\hline & GH5 & & & & & 8 & & 2 & & & 2 \\
\hline & GH6 & & & & & 8 & & & & & 6 \\
\hline & GHIO & & & & & 16 & & & 2 & & 7 \\
\hline & $\mathrm{GHII}$ & & & & & 4 & & & & & 1 \\
\hline \multirow[t]{6}{*}{$\mathrm{N}$-terminal domain } & CBM_2 & 7 & 8 & & & & & 2 & & & \\
\hline & CBM_3 & & & & & & & & & & \\
\hline & CBM_X2 & & & & & & & & & & \\
\hline & CBM_9 & & & & & & & & & & \\
\hline & CBM_4_9 & & & 4 & & I & & & 2 & & \\
\hline & & \multicolumn{10}{|c|}{ C-Terminal domain } \\
\hline (B) Thermophiles & & GH5 & GH6 & GHIO & GHII & CBM_2 & CBM_3 & CBM_X2 & CBM_9 & CBM_4_9 & No CBM \\
\hline & GH5 & & & & & 3 & 2 & & & & \\
\hline & GH6 & & & & & 5 & I & & & & 3 \\
\hline & GHIO & & & & & 8 & I & & 2 & & 3 \\
\hline & GHII & & & & & 3 & & & & & \\
\hline \multirow[t]{5}{*}{$\mathrm{N}$-terminal domain } & CBM_2 & 1 & 1 & & & & & & & & \\
\hline & CBM_3 & & & & & & & & & & \\
\hline & CBM_X2 & & & & & & & & & & \\
\hline & CBM_9 & & & & & & & & & & \\
\hline & CBM_4_9 & & & 2 & & & & & 2 & & \\
\hline
\end{tabular}




\section{Asymmetric amino acid substitutions in glycoside hydrolases}

We wanted to understand amino acid mesophilic Actinobacteria. This would help identify amino acid substitutions that may contribute to thermostability of glycoside hydrolases. For each glycoside hydrolase family, we aligned only the hydrolase domains of orthologs from mesophilic and thermophilic organisms identified earlier (Table 2). We calculated the frequencies of all amino acid substitutions between mesophilic and thermophilic homologs at every position and identified the statistically significant asymmetric amino acid substitutions (Table 4). The data revealed 41 pairs of amino acid substitutions that are asymmetric between the homologs from thermophilic and mesophilic Actinobacteria. Certain amino acid preferences in the thermophiles were specific to the glycoside hydrolase family, while other amino acid preferences were independent of the glycoside hydrolase family. For example, thermophilic enzymes from GH6, GH10, and GH11 families showed preferences for alanine over glycine. Similarly, thermophilic proteins showed preference for aspartate over thermolabile serine and threonine residues. There was also a biased preference for isoleucine over valine in thermostable homologs. Overall, the data provide several new targets for genetically engineering higher thermostability in glycoside hydrolases ${ }^{20}$ (Table 4).

Table 4 Asymmetric amino acid substitution patterns in glycoside hydrolases

\begin{tabular}{|c|c|c|c|c|c|c|c|c|c|c|c|c|c|c|c|c|}
\hline & & \multicolumn{15}{|c|}{ Amino acid in the thermophilic homologs } \\
\hline & & $\mathbf{A}$ & D & $\mathbf{E}$ & $\mathbf{F}$ & $\mathbf{H}$ & $\mathbf{I}$ & $\mathbf{K}$ & $\mathbf{L}$ & $\mathbf{N}$ & $\mathbf{P}$ & $\mathbf{Q}$ & $\mathbf{S}$ & $\mathbf{T}$ & $\mathbf{v}$ & $\mathbf{Y}$ \\
\hline \multirow{13}{*}{$\begin{array}{l}\text { Amino } \\
\text { acid in the } \\
\text { mesophilic } \\
\text { homologs }\end{array}$} & A & & C74 & C62 & Al00 & $\mathrm{BI} 00$ & & & & $\mathrm{C} 77$ & $\mathrm{BI} 00$ & $\begin{array}{l}\text { C6I, } \\
\text { B68 }\end{array}$ & & & & $\mathrm{BI} 00$ \\
\hline & $\mathrm{D}$ & B92 & & & & & & & & B93 & & & & & & \\
\hline & $\mathrm{E}$ & & A62 & & & & & & & & & & & & & \\
\hline & $\mathrm{F}$ & & & & & & & & & & & & & & & C65 \\
\hline & G & $\begin{array}{l}\text { B88, C67, } \\
\text { D86, }\end{array}$ & $\mathrm{ClOO}$ & & & & & B9l & & & & & & & & \\
\hline & $\mathrm{K}$ & & & A86 & & & & & & & & & & & & Al00 \\
\hline & $\mathrm{L}$ & & & & & & A62 & & & & & & & & & \\
\hline & $N$ & B 100 & D9I & & & D92 & & & & & & & D84 & DI00 & $\mathrm{Cl} 100$ & \\
\hline & $\mathrm{R}$ & & & $\mathrm{C} 67$ & & & & B90 & & & & C59 & & & & \\
\hline & $\mathrm{S}$ & D86 & $\begin{array}{l}\text { B77, } \\
\text { C67 }\end{array}$ & & & & & $\mathrm{Cl} 00$ & & & & C64 & & D85 & & \\
\hline & $\mathrm{T}$ & & $\begin{array}{l}\text { A80, } \\
\text { C75 }\end{array}$ & & & & $\begin{array}{l}\text { B100, } \\
\text { C78 }\end{array}$ & & & & $\mathrm{BI} 00$ & & & & B88 & D 100 \\
\hline & $\mathrm{V}$ & B80 & & & & & $\begin{array}{l}\text { A60, } \\
\text { C56 }\end{array}$ & & D77 & & & & & & & \\
\hline & $Y$ & A 100 & & & & & & & & & & & & & & \\
\hline
\end{tabular}

Standard single letter amino acid code is use to represent amino acids. Data are represented with a letter followed by a number, where A represents GH5, B represents $\mathrm{GH} 6, \mathrm{C}$ represents GHI0, D represents $\mathrm{GHI}$, and numbers represent the percentage of occurrence of the particular substitution. Only statistically significant $(p<0 . I)$ asymmetric substitutions are shown.

\section{Conclusion}

We analyzed the predicted proteomes of 69 sequenced Actinobacteria and identified homologs of 20 glycoside hydrolase families associated with plant cell wall degradation. Some glycoside hydrolase families were well represented across the phylum, while a few families were not represented in any of the Actinobacteria we analyzed. The glycoside hydrolases appear to have a constrained domain architecture that likely determines their stability, functioning, and interaction with substrates. Certain carbohydrate binding modules found fused to the glycoside hydrolases were only associated with thermophilic Actinobacteria. Finally, glycoside hydrolases from thermophilic Actinobacteria showed preferences for certain amino acid substitutions over their mesophilic counterparts. Overall, our data provide new insights into glycoside hydrolases in Actinobacteria and provide a basis for genetically enhancing the stability of glycoside hydrolases towards industrial applications.

\section{Acknowledgements}

This research was supported by startup funds provided to RDB by the University of Arkansas."KAT acknowledges support from the National Science Foundation Research Experience for Undergraduates program through the University of Arkansas REU Site (DBI-1063067)".

\section{Conflict of interest}

The author declares no conflict of interest.

\section{References}

1. Solomon BD. Biofuels and sustainability. Ann $N$ Y Acad Sci. 2010;1185:119-134.

2. Pothiraj C, Kanmani P, Balaji P. Bioconversion of lignocellulose materials. Mycobiology. 2006;34(4):159-165. 
3. Peralta-Yahya PP, Keasling JD. Advanced biofuel production in microbes. Biotechnol J. 2010;5(2):147-162.

4. Clark JH, Luque R, Matharu AS. Green chemistry, biofuels, and biorefinery. Annu Rev Chem Biomol Eng. 2012;3:183-207.

5. Cragg SM, Beckham GT, Bruce NC, et al. Lignocellulose degradation mechanisms across the Tree of Life. Curr Opin Chem Biol. 2015;29:108119.

6. Blumer-Schuette SE, Brown SD, Sander KB, et al. Thermophilic lignocellulose deconstruction. FEMS Microbiol Rev. 2014;38(3):393448 .

7. Bhalla A, Bansal N, Kumar S, et al. Improved lignocellulose conversion to biofuels with thermophilic bacteria and thermostable enzymes. Bioresour Technol. 2013;128:751-759.

8. Ventura M, Canchaya C, Tauch A, et al. Genomics of Actinobacteria tracing the evolutionary history of an ancient phylum. Microbiol Mol Biol Rev. 2007;71(3):495-548.

9. McCarthy AJ, Williams ST. Actinomycetes as agents of biodegradation in the environment-a review. Gene. 1992;115(1-2):189-192.

10. Ward AC, Bora N. Diversity and biogeography of marine Actinobacteria Curr Opin Microbiol. 2006;9(3):279-286.

11. Ohm RA, Riley R, Salamov A, et al. Genomics of wood-degrading fungi. Fungal Genet Biol. 2014;72:82-90.

12. Bruins ME, Janssen AE, Boom RM. Thermozymes and their applications: a review of recent literature and patents. Appl Biochem Biotechnol. 2001;90(2):155-186.
13. Kumar S, Nussinov R. How do thermophilic proteins deal with heat? Cell Mol Life Sci. 2001;58(9):1216-1233.

14. Cantarel BL, Coutinho PM, Rancurel C, et al. The Carbohydrate-Active EnZymes database (CAZy): an expert resource for Glycogenomics. Nucleic Acids Res. 2009;37(Database issue):D233-D238.

15. Altschul SF, Gish W, Miller W, et al. Basic local alignment search tool. $J$ Mol Biol. 1990;215(3):403-410.

16. Marchler-Bauer A, Derbyshire MK, Gonzales NR, et al. CDD:NCBI's conserved domain database. Nucleic Acids Res. 2015;43(Database issue):D222-226.

17. Takami H, Takaki Y, Chee GJ, et al. Thermoadaptation trait revealed by the genome sequence of thermophilic Geobacillus kaustophilus. Nucleic Acids Res. 2004;32(21):6292-6303.

18. Higgins, Desmond G, Sharp PM. CLUSTAL: A Package for Performing Multiple Sequence Alignment on a Microcomputer. Gene. 1988;73(1):237-244.

19. Larkin MA, Blackshields G, Brown NP, et al. Clustal W and Clustal X Version 2.0. Bioinformatics. 2007;23(21):2947-2948.

20. Gao SJ, Wang JQ, Wu MC, et al. Engineering hyperthermostability into a mesophilic family 11 xylanase from Aspergillus oryzae by in silico design of N-terminus substitution. Biotechnol Bioeng. 2013;110(4):1028-1038. 